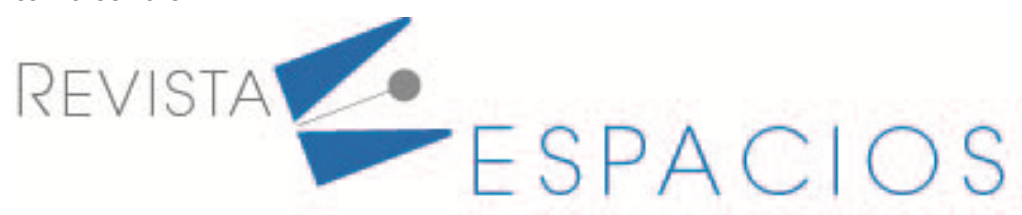

\title{
Estrategias adaptativas en comunidades rurales debido a la variabilidad climática
}

\section{Adaptive strategies in rural communities due to climate variability}

\author{
ESPINOSA ROMERO, Ana Patricia ${ }^{1}$ \\ ROSADO VEGA, Jairo Rafael ${ }^{2}$ \\ RODRÍGUEZ MIRANDA, Juan Pablo ${ }^{3}$
}

\begin{abstract}
Resumen
Esta investigación se realizó para identificar las relaciones causales del impacto de la variabilidad climática y las estrategias adaptativas en comunidades rurales, mediante un estudio de caso en el Resguardo indígena wayuu "El Pasito", en La Guajira, Colombia. Se analizó el periodo climático comprendido entre los años 2010 y 2016. Los resultados evidenciaron que la comunidad indígena El Pasito aplica estrategias adaptativas, principalmente frente a escenarios de riesgo por variabilidad climática, que intentan disminuir la vulnerabilidad del sistema productivo. Resultados estos que permitieron concluir que existe una marcada influencia negativa de la variabilidad climática sobre el sistema de producción ovino-caprina ancestralmente aplicado en la comunidad indígena wayuu El Pasito. Además, se concluyó que la comunidad El Pasito es resiliente y ha implementado estrategias adaptativas por conocimiento local y en algunos casos por transferencia tecnológica.

Palabras clave: desarrollo rural, adaptación, producción ovino-caprina
\end{abstract}

\begin{abstract}
This research was carried out to identify the causal relationships of the impact of climate variability and adaptive strategies in rural communities, through a case study in the Wayuu indigenous reservation "El Pasito", in La Guajira, Colombia. The climatic period between 2010 and 2016 was analyzed. The results showed that the El Pasito indigenous community applies adaptive strategies, mainly in the face of risk scenarios due to climate variability, which try to reduce the vulnerability of the productive system. These results allowed us to conclude that there is a marked negative influence of climate variability on the sheep-goat production system ancestrally applied in the El Pasito Wayuu indigenous community. In addition, it was concluded that the El Pasito community is resilient and has implemented adaptive strategies for local knowledge and in some cases for technology transfer.

Keywords: rural development, adaptation, sheep-goat production
\end{abstract}

\section{Introducción}

El Banco Interamericano de Desarrollo destaca el enfoque de territorialidad en el desarrollo rural sostenible con el fin de promover la economía rural. Este concepto, desde el enfoque territorial busca integrar las dimensiones

\footnotetext{
${ }^{1}$ Docente. Facultad de Ingenierías. Universidad de La Guajira. Correo electrónico: apespinosa@uniguajira.edu.co

2 Profesor Titular. Facultad de Ingeniería. Universidad de La Guajira. Correo electrónico: jrosado@uniguajira.edu.co

${ }^{3}$ Profesor Titular. Facultad del Medio Ambiente y Recursos Naturales. Universidad Distrital Francisco José de Caldas. Correo electrónico: jprodriguezm@udistrital.edu.co
} 
económicas, sociocultural, ambiental, político - institucional, con el objetivo de promover el bienestar de la sociedad rural de modo que pueda contribuir al desarrollo general de la población (Sepúlveda, et al., 2003, p.160).

Según posturas que enmarcan la concepción de desarrollo rural en Latinoamérica, autores como Farah y Pérez (2003), afirman que el desarrollo rural se orienta hacia una visión de lo regional y la sostenibilidad, no sólo de recursos naturales, sino también económica, política, social y cultural, e igualmente incorpora el concepto de empoderamiento de las comunidades campesinas, la perspectiva de equidad de género y de la participación de los distintos actores sociales en los diferentes procesos y proyectos de desarrollo. En este sentido, Rojas (2008), plantea la importancia de incorporar el análisis de la identidad territorial y la multiescalaridad de los territorios, dando lugar a modelos de Desarrollo Rural con Enfoque Territorial.

De acuerdo con Herrera (2013), la definición de desarrollo rural remite a una condición deseable de bienestar para los habitantes de un territorio considerado - según ciertos parámetros y calificaciones - como rural. "Cuando el desarrollo no se refiere al conjunto de una sociedad, sino que se centra en las áreas rurales en las que se pretende mejorar el nivel de vida de su población, a través de procesos de participación local y mediante la potenciación de sus recursos propios, éste se define como desarrollo rural" (Guzmán et al., 1999, citado por Herrera, 2013, p. 132).

Según Dixon, et al., (2001) los sistemas de producción agropecuaria toman en cuenta tanto la dimensión biofísica, como los aspectos socioeconómicos al nivel de finca, en donde se toman la mayoría de decisiones en cuanto a la producción agrícola y al consumo. El alcance de este enfoque radica en su capacidad de integrar los distintos tipos de análisis multidisciplinarios de la producción y de su relación con los aspectos biofísicos y socioeconómicos determinantes de un sistema de producción agropecuario. (p.17).

Scalone Echave (2007), por su parte, hace referencia al sistema de producción agropecuario, entendido como la forma en que el productor organiza la utilización de sus recursos en función de sus objetivos y necesidades, condicionado por factores externos de carácter socioeconómico y ecológico. Lo anterior, permite inferir que el concepto de sistemas de producción adquiere significado epistémico de acuerdo con el sector de producción al que se aplica, de allí que el concepto de producción rural se asocia con unidades de producción que se encuentran "atadas al devenir rural de sus habitantes" (INEGI, 1994, p.173), y a las actividades que allí se realizan en lo concerniente a lo agropecuario, forestal y/o agroindustrial, que contribuyen al desarrollo rural de una región.

Es importante anotar, que a través del efecto climático (sequías e inundaciones), los fenómenos de El Niño y La Niña, tienen un gran impacto socioeconómico en diferentes países, que está muy relacionado con la vulnerabilidad de las poblaciones y de los sectores productivos. (Montealegre \& Pabón, 2000 \& Arango et al., s.f.). Hay que mencionar, además, que el efecto climático registrado durante los fenómenos "La Niña" no es exactamente contrario al observado durante los fenómenos "El Niño", aunque si se observa una tendencia inversa en el efecto producido por ambos eventos (Montealegre, 2009, p.11).

Entre otras cosas, a nivel científico, existe un alto de grado de consenso en que el cambio climático exacerba la variabilidad climática generando (DNP, 2012): cambios inusuales en los rangos registrados de las variables climáticas y, variación en la frecuencia, duración e intensidad de los eventos climáticos extremos. Entendiendo por cambio climático la modificación a largo plazo de las condiciones meteorológicas medias a escala del planeta, debido a la variabilidad natural o como resultado de actividades humanas, que pueden representar una amenaza natural, como inundaciones, sequías, olas de frío o de calor, tormentas, etcétera (OMM, 2009; IPCC, 2007). 
Por ello, este manuscrito, tiene como objetivo, identificar las relaciones causales del impacto de la variabilidad climática y las estrategias adaptativas en comunidades rurales, mediante un estudio de caso en el Resguardo indígena "El Pasito", en Riohacha, La Guajira (Colombia).

\section{Desarrollo}

Ulloa (2008, 2012); Crate y Nuttall (2009) se han interesado por investigar la relación naturaleza-cultura, haciendo mención a que los fenómenos de cambios del clima involucran cambios ambientales con las distintas formas en que la cultura de diversos grupos sociales piensa y genera conocimientos relacionados con su entorno local. En relación con Echeverri (2009), las comunidades indígenas han venido sufriendo efectos directos del cambio climático global, aunados con otros factores que también afectan los modos de vida indígena, caracterizándose por la capacidad pluriactiva, que les permite adaptarse y hacer frente a múltiples cambios en su entorno.

Pinilla-Herrera et al. (2012), manifiestan la importancia de la participación de las comunidades locales en el planteamiento de estrategias de adaptación al cambio climático; de igual manera, destacan que los saberes locales establecen otra forma de conocimiento que se justifica en la experiencia y por esta razón, son conocimientos válidos, verificables y con credibilidad. Para la FAO (s.f.), los sistemas de producción pecuaria, son considerados como la estrategia social, económica y cultural más apropiada para mantener el bienestar de las comunidades, debido a que es la única actividad que puede simultáneamente proveer seguridad en el sustento diario, conservar ecosistemas y valores culturales y tradiciones. Además, afirma que la producción agrícola y ganadera de la región estará cada vez más influenciada por factores climáticos y plantean la necesidad de implementar nuevos métodos y herramientas que permitan incorporar la experiencia y el conocimiento local, haciendo énfasis en la riqueza de conocimientos tradicionales de los productores agropecuarios campesinos de muchas regiones del mundo, para enfrentar la variación y los riesgos del clima, lo cual es necesario para poder generar procesos de vinculación y transferencia de experiencias exitosas.

El proyecto realizado por la unidad de agroclima y sistemas de información (GRAS) del Instituto Nacional de Investigación Agropecuaria (INIA) de Uruguay, el Banco Mundial y la Universidad de Cornell de los Estados Unidos, sobre Vulnerabilidad al cambio climático en los sistemas de producción agrícola en América Latina y el Caribe: desarrollo de respuestas y estrategias, cuyo objetivo fue formular un plan de acción con recomendaciones para el desarrollo de respuestas y estrategias con el fin de contribuir a una mejor adaptación a los impactos de la variabilidad climática y la ocurrencia de eventos climáticos extremos en los sistemas de producción agrícola de Uruguay, obtuvo como resultado la identificación y priorización de tres opciones de respuesta: (a) sistema de información y soporte para la toma de decisiones; (b) gestión del agua; (c) seguros y otros instrumentos financieros para la gestión de riesgos (Giménez \& Lanfranco, 2012).

En este sentido, Ulloa (2012) orientó su investigación en torno al clima y sus cambios, como un proceso que involucra la relación del hombre-naturaleza y desde la perspectiva de las comunidades indígenas, analizó los conocimientos y saberes de las comunidades frente al cambio climático y sus contribuciones a los procesos de adaptación a nuevas condiciones climáticas. En efecto, afirma en su documento que los cambios climáticos se han dado históricamente y los pueblos indígenas han generado estrategias para enfrentarlos, teniendo en cuenta factores ligados a la cultura de cada comunidad local, como ritmos, calendarios y prácticas cotidianas ligadas a ciclos ambientales; igualmente, a factores de tipo ambiental e institucional (Ulloa, 2008).

Sostiene el Ministerio de Medio Ambiente y Desarrollo Sostenible (2015) que: "la suma de visiones desde el conocimiento ancestral de las comunidades indígenas y campesinas, será de gran importancia para lograr medidas de respuesta adaptativas fundamentadas en sus pobladores" (p. 131) por este motivo para este Ministerio "el conocimiento científico debe ser combinado con el conocimiento ancestral en temas relevantes 
como la información sobre la variabilidad climática y alertas tempranas" (p. 104). Por otro lado, Ifejika (2010), en estudio desarrollado en el distrito de Makueni, Kenia, analiza cómo los productores adaptan la producción ganadera a la variabilidad climática, obteniendo los datos a través de encuestas, encontrando que las enfermedades del ganado son los principales desafíos durante períodos de lluvia intensa y sequía extrema. Se identifican en el estudio que son pocas las respuestas ante estos eventos y recomiendan incorporar estrategias agropastoriles para mejorar la capacidad de adaptación.

Rubio y Roig (2017), en su estudio sobre impactos, vulnerabilidad y adaptación al cambio climático en los sistemas extensivos de producción ganadera en España, llegaron a la conclusión que la gestión del pastoreo, se convierte en la principal herramienta de adaptación a las transformaciones de la variabilidad climática. Otra de las estrategias es la predicción ajustada del comportamiento de los factores de producción de pastos a corto y mediano plazo (ej. precipitaciones y temperaturas en escala de semanas o meses). Por su parte, el estudio sobre experiencias de adaptación al cambio climático, conocimientos ancestrales, conocimientos contemporáneos y los escenarios cualitativos en los Andes (Perú), realizado por Torres Guevara (2015), describe las tecnologías apropiadas ancestrales para la variabilidad climática y el cambio climático. Una de ellas, sobre todo en épocas de sequía son las denominadas Waru waru, huachos, protección de bosques, huertas, uso de especies cultivadas nativas resistentes; y los reservorios hechos a base de champas de ichu en quebradas, empleadas para almacenamiento de agua. Adicionalmente, para la regulación del flujo hídrico, hacen uso de los surcos en sentido transversal a la pendiente cuando el año va a ser seco. Cuando se presenta el fenómeno del Niño, acuden a la técnica de bioindicadores (insectos, aves, plantas, etc.) y fases lunares con el objetivo de hacer seguimiento al clima, estas últimas reconociendo la cosmogonía de las culturas, modo de percepción y de observación, modo experimental del conocimiento tradicional, el respeto y cuidado por la naturaleza.

A nivel local, la Fundación Alpina y la Alcaldía de Riohacha (2014), realizaron un estudio cuyo propósito fue establecer estrategias para aportar al fortalecimiento y sostenibilidad de la producción ovino-caprina como actividad productiva ancestral Wayuu, que además es básica para la seguridad alimentaria de las comunidades, atendiendo las problemáticas que más los afectan, como es el alto índice de mortalidad del asentamiento. Aún más, cuando se encuentran frente a las consecuencias del cambio climático, que hacen cada vez más difícil la cría y levante ovino-caprino en las condiciones agroecológicas de La Guajira.

Por otra parte, se encuentra el estudio realizado por Salazar (2017), en donde se muestra el proceso para determinar la viabilidad y factibilidad en la creación de una granja dedicada a la producción de ovinos por medio de un cruzamiento con hembras criollas de la región y machos puros de la raza Katahdin, en un programa de cría, levante y ceba, en el municipio de Maicao en el departamento de La Guajira (Colombia) mediante sistemas de producción semi-intensivo en pastoreo de gramíneas. Para lograr este objetivo, se realizaron procesos correspondientes al análisis técnico, de mercado, legal, ambiental y financiero; lo cual arrojó como resultado principalmente una baja oferta en el mercado local.

Igualmente, el Ministerio de Ambiente y Desarrollo Sostenible (2013) de Colombia, explica que la labor productiva de los wayuu está vinculada económicamente al pastoreo, puesto que son un pueblo de pastores por naturaleza y dedican el mayor tiempo posible al cuidado de animales vacunos, caprinos, ovinos, mulares y caballares, los cuales integran a la cultura a partir de los primeros contactos con el hombre europeo en su territorio. La cría de ganado se constituye en la principal fuente de la economía tradicional, a cuyo valor patrimonial se integra un valor simbólico de gran efecto en el pago de compensaciones. De ahí que las especies de ganado representen un recurso social de gran importancia para su convivencia pacífica.

De acuerdo con la revisión realizada en las investigaciones previas, se puede decir que el departamento de La Guajira está expuesto a riesgos asociados a la variabilidad climática y el cambio climático, así se expresa en el Plan Integral de Cambio Climático (2015), entre ellos se encuentran: "el déficit hídrico, las inundaciones y 
deslizamientos" (Cámara de Comercio de Bogotá, 2015); cambios significativos en las condiciones climáticas, los cuales afectan de manera directa a la seguridad alimentaria (en este caso a las comunidades indígenas de la etnia Wayuu); por lo tanto, la frecuencia de fenómenos climáticos extremos e intensos como las sequías y las irregularidades crecientes en los regímenes de lluvias puede repercutir en los medios de subsistencia de la etnia Wayuu.

En síntesis, las principales investigaciones han avanzado en variables fundamentales para este estudio tales como: la variabilidad climática es el principal efecto del cambio climático; la alteración de las variables climáticas disminuye la productividad de los sistemas pecuarios, con consecuencias adversas para la sostenibilidad de las comunidades rurales; la mayor concentración productiva de caprinos y ovinos de Colombia, se encuentra en la Costa Atlántica, principalmente en el departamento de La Guajira; la producción ovina y caprina constituyen el principal medio de subsistencia de familias campesinas en diferentes zonas de Colombia; los conocimientos tradicionales son fundamentales para la implementación de estrategias adaptativas exitosas frente a la variabilidad climática; la frecuencia de fenómenos climáticos extremos puede repercutir en los medios de subsistencia de la etnia Wayuu.

\subsection{Adaptación}

La adaptación, hace referencia generalmente al proceso, acción o resultado en un sistema en orden que pueda enfrentar, manejar o ajustarse a alguna condición cambiante, estrés, amenaza, riesgo u oportunidad (Smit \& Wandel, 2006), en este caso los asociados con la variabilidad climática. El Panel Intergubernamental sobre Cambio Climático (IPCC, p. 76), define adaptación como: "El ajuste en los sistemas naturales o humanos como respuesta a estímulos climáticos actuales o esperados, o sus impactos, que reduce el daño causado y que potencia las oportunidades benéficas".

Las sociedades no son ni han sido sujetos pasivos frente a las amenazas naturales. Dichos caminos sociales y culturales se manifiestan en hábitos, costumbres, comportamientos, tradiciones y prácticas específicas, que son parte de la cotidianidad en ciertas sociedades o ciertos sectores sociales. Estos hábitos, costumbres, comportamientos, tradiciones y prácticas desarrollados frente a las amenazas naturales o eventos climáticos, son las construcciones culturales a las que denominamos estrategias adaptativas (Quintero-Angel \& CarvajalEscobar, 2010). Acorde con lo anterior, Steward señala que: "si bien las culturas tratan de perpetuarse a lo largo de los siglos, las culturas que enfrentan ambientes diferentes han tenido que cambiar enormemente y estos cambios se pueden trazar a partir de las nuevas adaptaciones requeridas por la cambiante tecnología y los acomodos productivos" (1973, p. 37), en consecuencia, la adaptación al medio constituye un importante proceso creativo (p. 5).

Las estrategias de adaptación son culturalmente construidas en sus adaptaciones al medio, cambiantes y dinámicas (Ulloa, 2011; Acosta, 2006), en general, son una amplia gama de acciones de ajuste en respuesta a o en anticipación de condiciones cambiantes en el entorno, que pueden involucrar cambios o procesos sociales, la ingeniería, cambios en prácticas agrícolas, cambios tecnológicos, uso más eficiente de recursos hídricos, entre otros (Heller \& Mani, 2002; FAO 2003 \& UNEP, 2003, citado por Quintero-Angel \& Carvajal-Escobar, 2010), y dependen del manejo y conocimiento culturales del ambiente que se hayan alcanzado, así como del grado de dependencia o independencia conseguida con respecto a los recursos disponibles. Por ello, con frecuencia son desarrolladas a escala local y, en algunos casos, regional (Acosta, 2006).

\subsection{Vulnerabilidad}

El Intergovernmental Panel on Climate Change IPCC, en 2007, definió a la vulnerabilidad como el grado en que un sistema es susceptible a, o incapaz de afrontar los efectos adversos del cambio y la variabilidad climática y sus 
extremos. Por su parte, en 2014, la define como la propensión o predisposición a ser afectado negativamente. La vulnerabilidad comprende una variedad de conceptos y elementos que incluyen la sensibilidad o susceptibilidad al daño y la falta de capacidad de respuesta y adaptación (IPCC, 2014).

La vulnerabilidad es entendida como un fenómeno complejo que se genera en el cruce de las dimensiones ambientales, sociales y económicas (Mussetta \& Barrientos, 2015). Por lo tanto, con base en lo señalado por Basualdo, et al (2015), la vulnerabilidad en este contexto se define como el grado de susceptibilidad de un sistema productivo de sufrir daños por la ocurrencia de un fenómeno adverso. Es la medida en que un sistema es incapaz de afrontar los efectos negativos de cada amenaza climática particular, incluyendo la variabilidad climática y los fenómenos extremos, está dada por las características y las circunstancias del sistema, que lo hacen más o menos susceptible a los efectos dañinos que podría producir una amenaza particular.

\subsection{Conocimiento local}

En este sentido, para comprender las transformaciones ambientales es necesaria la introducción y el reconocimiento de nociones y conocimientos locales, lo cual implica partir de valores, percepciones y experiencias individuales, y relaciones sociales, morales y éticas que plantean diversas culturas frente a esas transformaciones (Ulloa, 2012). Acosta, et al (2011), señalan que los saberes y prácticas locales que han existido a través de la historia de las civilizaciones, son una evidencia de los vínculos entre los sistemas sociales y ecológicos como base del bienestar humano. En este sentido, plantean que: "Los saberes locales están constituidos por esquemas y modelos complejos de información que aportan soluciones prácticas, nacidas de las condiciones locales como respuestas adaptativas acompasadas con los tiempos de los procesos biofísicos esenciales" (Acosta et al., 2011, p. 2).

En general, los conocimientos locales hacen referencia al saber y a las habilidades y filosofías que han sido desarrolladas por sociedades de larga historia de interacción con su medio ambiente (UNESCO, 2015); provienen de la observación cotidiana. Estos se basan en la experiencia de generaciones pasadas con formas de vida, sistemas productivos y ecosistemas naturales; adaptándose a los nuevos cambios tecnológicos y socioeconómicos del presente (Johnson, 1992; Montecinos, 1999). El conocimiento local se refleja en las tradiciones y prácticas ancestrales de un determinado grupo social (familia, etnia, comunidad) y representa un conjunto de respuestas adaptativas a situaciones o problemas que resultan de la interacción con el medio biofísico.

Según los actores entrevistados, la zona del resguardo indígena El Pasito, es una zona inundable, principalmente en los alrededores del área comunitaria o del caserío como tal, lo cual ha generado problemas manifiestos en el proceso de producción ovina-caprina. Específicamente durante los últimos diez años, la variabilidad climática observada en el área ha provocado problemas en el proceso productivo, específicamente durante las lluvias de los años 2010 y 2011, con valores anuales de $1560,5 \mathrm{~mm}$ y 1571,7 mm respectivamente (IDEAM, 2018b), y cuyos niveles de precipitación son significativamente diferentes $(H=16,91 ; p=0,015)$, de los años 2013, 2014 y 2015, los cuales son los de menor precipitación, estableciéndose como los años de sequía extrema, dentro del periodo estudiado (2009 - 2016); los entrevistados manifestaron que se presentó un efecto negativo por el exceso de agua e inundaciones registradas, el cual consistió en el aislamiento de los animales durante este periodo, es decir, se perdió la conectividad entre el resguardo y las zonas aledañas, lo que ocasionó que los animales no pudieran salir a pastorear, y la muerte de muchos de ellos por inanición.

Aunado a lo anterior, se destaca que la condición opuesta, es decir, la sequía extrema, también ha traído efectos negativos en la producción de ganado ovino-caprino, especialmente en los últimos años del periodo estudiado, de los cuales se infieren a los años 2014 y 2015, cuyas precipitaciones fueron las de menor valor anual, con 260,5 $\mathrm{mm}$ y $152 \mathrm{~mm}$ respectivamente (IDEAM, 2018), como aquellos con mayor representación del efecto sequía; los 
entrevistados manifestaron que "los últimos veranos han sido fuertes", destacan muerte de animales por inanición, ya que hubo escasez de pasto.

La sequía ha generado afectaciones a la actividad ovino-caprina, dado que la escasez de agua implica que el ganado se aleje cada vez más de las rancherías en busca del líquido, es así que en épocas de sequías largas se presentan muertes masivas de los animales ocasionando pobreza y hambre, debido a que el pueblo indígena solo cuenta con su territorio y su mano de obra para su supervivencia (Asociación de Autoridades Tradicionales de La Guajira AKALINJIRA WA, Ministerio del interior, 2014). La Tabla 1 resume los impactos asociados a la variabilidad climática en el sistema de producción ovino- caprina de El Pasito.

Tabla 1

Impactos de la variabilidad climática

\begin{tabular}{|c|c|c|}
\hline Evento climático & Tipo & Impactos \\
\hline \multirow{3}{*}{ Lluvias intensas } & Ambientales & $\begin{array}{l}\text { Inundaciones. } \\
\text { Aumento de enfermedades. }\end{array}$ \\
\hline & Económicos & $\begin{array}{c}\text { Muerte de animales. } \\
\text { Disminución de los ingresos. }\end{array}$ \\
\hline & Sociales & $\begin{array}{l}\text { Pérdida de conectividad con zonas aledañas. } \\
\text { Aumento de la pobreza. } \\
\text { Fragilidad de los mecanismos de resiliencia. } \\
\text { Adopción de nuevos roles }{ }^{4} \text {. }\end{array}$ \\
\hline \multirow{3}{*}{ Sequía extrema } & Ambientales & $\begin{array}{l}\text { Baja productividad del suelo. } \\
\text { Escasa disponibilidad de agua. } \\
\text { Deforestación. } \\
\text { Desertización. } \\
\text { Altas temperaturas (aumento de estrés animal). } \\
\text { Desaparición de algunas especies (como el mamoncillo) y baja } \\
\text { productividad de otras esenciales para su alimentación. }\end{array}$ \\
\hline & Económicos & $\begin{array}{c}\text { Escasez de forraje. } \\
\text { Muerte de animales. } \\
\text { Disminución de los ingresos. }\end{array}$ \\
\hline & Sociales & $\begin{array}{l}\text { Debilitamiento de los mecanismos de resiliencia, por la dificultad } \\
\text { para predecir el clima. } \\
\text { Alteración de los ciclos de siembra. } \\
\text { Cambio en los hábitos de consumo. } \\
\text { Aumento de la pobreza. }\end{array}$ \\
\hline
\end{tabular}

Fuente: Autores

Analizando la variabilidad ambiental dentro del periodo estudiado, se pudo observar que a inicios del periodo, se presentaron las lluvias intensas, mientras que a finales del periodo se presentaron sequías extremas, por lo que se puede destacar eventos extremos de variación climática, que según los entrevistados son una de las causas por la cual antes de estas eventualidades, la cantidad de ganado era mayor que la que presentan actualmente, sin quitarle mérito a otros factores que también manifestaron los entrevistados, como la mudanza de algunas familias cuyo ganado también fue retirado de la comunidad (lo que explica solo cinco corrales activos), así como también el robo de animales del rebaño. Por lo anterior, la producción se ha visto afectada de manera

\footnotetext{
${ }^{4}$ Ante las debilidades de la actividad económica de producción ovina- caprina los hombres han aprendido a tejer, actividad que culturalmente era exclusiva de las mujeres.
} 
multifactorial, principalmente por la variabilidad climática y los robos, estos últimos un fenómeno que, aunque siempre presente, se ha potenciado en los últimos años (2016-2018).

Como estrategias aplicadas antes o durante las épocas climáticas comunes (sequía y lluvia), se realiza la siembra de pasto a finales de la sequía, puesto que las lluvias son esperadas como un momento positivo para la producción ovino-caprina, en vista de que el forraje crece y abunda durante y posterior a las precipitaciones. De hecho, durante las lluvias, manifiestan que, en ocasiones cuando hay insumos para hacerlo, realizan el marcaje de los animales (perforación de orejas), ya que la producción suele aumentar en esta época por la abundancia de pasto.

Los entrevistados manifestaron que los corrales permanecen en la misma ubicación dentro del resguardo, solo se mueven cuando las excretas son excesivas (largos periodos de tiempo), y de ser el caso, son reubicados a un lado de la localización anterior. Asimismo, expresaron que hicieron canales para la salida del agua de los corrales que se inundaban con las lluvias. Por otro lado, informan que: "Cuando hay mucha lluvia los chivos se dejan en la parte alta; los que quedan aislados los trasladamos para la parte alta". (E2, comunicación personal, diciembre de 2018).

Por su parte, se observó la adecuación del techo de los corrales mediante el uso de materiales no tradicionales para la etnia wayuu, como las láminas de zinc; así como el mejoramiento de la sanidad animal, para lo cual se ayudan con la asistencia técnica. Por todo lo anterior, se evidenció que existen estrategias adaptativas per se, para la variabilidad climática en el resguardo indígena El Pasito, lo que en cierta forma responde a las manifestaciones de pérdida de ganado en los últimos años, con cambios extremos del clima, desde inundaciones en los años 2010-2011 y sequías intensas en los años 2014-2015, ya que la estrategia no es suficiente para enfrentar los efectos climáticos drásticos que se han experimentado sobre todo en los últimos diez años. Las estrategias de adaptación empleadas por los productores wayuu de El Pasito, en respuesta o en anticipación a los eventos climáticos de lluvia y sequía, se resumen en la tabla 2.

Tabla 2

Estrategias de adaptación de El Pasito

\begin{tabular}{|c|c|c|c|}
\hline \multirow{2}{*}{\multicolumn{2}{|c|}{ Estrategias de adaptación }} & \multicolumn{2}{|c|}{ Evento climático } \\
\hline & & \multirow{2}{*}{$\frac{\text { Lluvia }}{X}$} & \multirow[t]{2}{*}{ Sequía } \\
\hline \multirow{5}{*}{ En respuesta } & Adecuación de canales en corrales & & \\
\hline & Ubicación del ganado en zonas altas & $x$ & \\
\hline & Siembra de pasto & & $x$ \\
\hline & Identificación o marcaje del ganado & $\mathrm{X}$ & \\
\hline & Mejoramiento de la sanidad animal & $x$ & \\
\hline \multirow[b]{3}{*}{ En anticipación } & Siembra de pasto & $x$ & \\
\hline & Selección de semillas & $\mathrm{X}$ & \\
\hline & $\begin{array}{c}\text { Adecuación del techo de los corrales } \\
\text { mediante el uso de materiales no } \\
\text { tradicionales }\end{array}$ & $\mathrm{x}$ & $x$ \\
\hline
\end{tabular}

Fuente: Autores

\section{Conclusiones}

La comunidad indígena El Pasito aplica estrategias adaptativas, sobre todo, frente a escenarios de riesgo por variabilidad climática que intentan disminuir la vulnerabilidad del sistema productivo. Muchas de ellas tienen un impacto positivo en el manejo de la crisis y se destacan la siembra de pastos previa a la sequía; canales para el desagüe de los corrales en épocas lluviosas; adecuación del techo de los corrales mediante el uso de materiales 
no tradicionales para la etnia wayuu, como las láminas de zinc; traslado del rebaño a zonas altas y mejoramiento en el manejo de la sanidad animal (ej. aplicación de vacunas). Esto quiere decir que han implementado procesos, pero no son conscientes de que son estrategias adaptativas.

Los resultados permiten afirmar que existe una influencia negativa de la variabilidad climática en el periodo estudiado sobre la producción de ganado ovino-caprino ancestralmente aplicada en la comunidad indígena wayuu El Pasito. Así se concluye que la comunidad El Pasito es resiliente y ha implementado estrategias adaptativas por conocimiento local y en algunos casos por transferencia tecnológica, para disminuir la vulnerabilidad a los cambios del clima. Además, se concluye que las estrategias adaptativas son elementos constitutivos de la sociedad, es decir, son parte de la adaptación que las sociedades han llevado a cabo con el medio que les rodea y del tipo de relaciones que han desarrollado tras haber vivido en condiciones de riesgo por los fenómenos climáticos (Maffesoli, 2005, citado por Acosta 2006, p. 40). Así, la adaptación a la variabilidad climática constituye un proceso creativo y requiere estar dispuesto a cambiar de paradigmas.

\section{Referencias}

Acero, V. (2014). El bienestar animal en sistemas productivos de ovinos-caprinos en Colombia. 1Asociación Nacional de Médicos Veterinarios de Colombia (amevec), 57-62.

Acosta, R.; Boixo, J.; Lebrato, J.; Montes, C.; et al. (2011). Manifiesto Sobre Saberes Locales de La Universidad Internacional de Andalucía En: 6o Foro Internacional Saberes Para El Cambio. Sevilla (España). Rectorado UNIA. (Fecha de consulta: 20 de octubre de 2015). Disponible en: http://asos.unia.es/images/PDF/6ForoManifiesto.pdf

Acosta, V. (2006). Estrategias adaptativas y amenazas climáticas. Más allá del Cambio Climático: las dimensiones psicosociales del cambio ambiental global.

Altieri, A. \& Nicholls, C. (2008). Los impactos del cambio climático sobre las comunidades campesinas y de agricultores tradicionales y sus respuestas adaptativas. Department of environmental Science, Policy and Management, University of California. Revista Agroecología, Vol. 3.

Arango, C.; Dorado, J.; Guzmán, D. \& Ruíz, J. (s.f.). Variabilidad climática de la precipitación en Colombia asociada al ciclo El Niño, La Niña-Oscilación del Sur (ENSO). Grupo de Modelamiento de Tiempo, Clima y Escenarios de Cambio Climático Subdirección de Meteorología - IDEAM.

Asociación de Autoridades Tradicionales de La Guajira AKALINJIRA WA \& Ministerio del interior (2014). Plan de salvaguarda wayuu capítulo Riohacha. Riohacha, Colombia. 338 p.

Basualdo, A., Berterretche, M., \& Vila, F. (2015). Inventario y características principales de los mapas de riesgos para la agricultura disponibles en los países de América Latina y el Caribe (No. IICA P40). IICA, San José (Costa Rica). ISBN: 978-92-9248-589-4.

Benavides, M. \& Gomez-Restrepo, C. (2005). Métodos en investigación cualitativa: triangulación. rev.colomb.psiquiatr. [online]. Vol.34, n.1, pp. 118-124. ISSN 0034- 7450. Bogotá, Jan./Mar.

Bonilla, E.; Hurtado, J. \& Jaramillo, C. (2009). La investigación: Aproximaciones a la del conocimiento científico. Alfaomega, Bogotá.

Borroto, Á.; Pérez, R.; Mazorra, C.; Carmenate, A.; Barrabí, M. \& Arencibia Á. (2011). Caracterización socioeconómica y tecnológica de la producción ovina en Ciego de Ávila, región Central de Cuba (Parte I). Pastos y Forrajes, 34(2), 199-210. 
Caicedo, J., Avila, M. \& Cubides, J. (2016). Enfermedades respiratorias de vías aéreas bajas en ovinos, impacto regional, principales etiologías infecciosas y métodos de diagnóstico. Revista Zoociencia, 3(1), 25-32.

Calvo-Solano, O. D., Quesada-Hernández, L., Hidalgo, H., \& Gotlieb, Y. (2018). Impactos de las sequías en el sector agropecuario del Corredor Seco Centroamericano. Agronomía Mesoamericana, 29(3), 695-709. Obtenido de http://dx.doi.org/10.15517/ma.v29i3.30828

Cámara de Comercio de Bogotá. (2015). Formulación e implementación del Plan Integral de Cambio Climático Para el departamento de La Guajira. Bogotá: Corporación Ambiental Empresarial - CAEM.

Carabalí, A. (2014). Por una antropología en territorio: dinámicas territoriales, morfologías sociales y configuraciones culturales entre los indígenas Wayuu (Colombia). Tesis doctoral, Universidad de los Andes. Mérida - Venezuela.

Castellanos, M. \& Carabalí, A. (2014). El delta del río Ranchería una mirada biofísica y social antes de la represa. Universidad de La Guajira. ISBN. 978-958-8530-24-6.Riohacha, Colombia. 160 pp.

Caviedes, C. (2001). The Niño in History: Storming Through the Ages. University Press of Florida. ISBN. 978081-30209-9-0. 279 pp.

Centro de Pensamiento en Desarrollo Rural (CPDR). (2016). Desarrollo rural con enfoque territorial para la paz en Colombia. Universidad Nacional de Colombia. 254 pp.

Colombia. Ministerio de Ambiente y Desarrollo Sostenible, 2015. (2015). Plan Integral de Gestión del Cambio Climático Territorial del Departamento de Magdalena. Bogotá: UT CAEM-E3 (consultor).

Corporación Autónoma Regional de La Guajira (Corpoguajira). (2011). Atlas ambiental del departamento de La Guajira. ISBN. 978-958-99258-1-2. 191 pp.

. (2018). en Plan Integral de Cambio Climático del departamento de La Guajira 2018 - 2030. Riohacha.

Corpoguajira, Parques Nacionales Naturales de Colombia \& Conservación Internacional Colombia. (2011). Tomo 2: Plan de Ordenamiento de la Cuenca del Río Ranchería. Diagnostico general (Versión Final). República de Colombia. $567 \mathrm{pp}$.

Corporación Colombiana de Investigación Agropecuaria. (28 de junio de 2018). AGROSAVIA. Recuperado el 24 de abril de 2019, de http://www.corpoica.org.co/noticias/generales/ovinos-guajira/

Corporación Colombiana de Investigación Agropecuaria- CORPOICA. (2016). Plan de Manejo Agroclimático Integrado del Sistema Productivo de Ganadería Ovino-Caprina. Uribia -La Guajira.

Crate, S. \& Nuttall, M. (2009). Anthropology and climate change. Walnut Creek, CA.: Left Coast Press.

Cuartas, C.; Naranjo, J.; Tarazona, A.; Murgueitio, E.; Chará, J.; Ku Vera, J. \& Barahona,R. (2014). Contribution of intensive silvopastoral systems to animal performance and to adaptation and mitigation of climate change. Revista Colombiana de Ciencias Pecuarias, 27 (2), 76-94.

De La Rocque, S. (2008). Introducción. In: Cambio climático: influencia en la epidemiología y las estrategias de control de enfermedades animales. Rev. Sci. Tech. Off. Int. Epiz 27 (2), 307-308. 
Departamento Nacional de Planeación (DNP). (2012). ABC: Adaptación bases conceptuales. Marco conceptual y lineamientos del Plan Nacional de Adaptación al Cambio Climático (PNACC). Departamento Nacional de Planeación (DNP) y Subdirección de Desarrollo Ambiental Sostenible (SDAS). Manuscrito borrador. 75 pp.

Dixon, J., Gulliver, A., y Gibbon, D. (2001). Sistemas de Producción Agropecuaria y Pobreza: Cómo mejorar los medios de subsistencia de los pequeños agricultores en un mundo cambiante. Roma y Washington D.C: FAO y Banco Mundial. Obtenido de http://www.fao.org/3/a-ac349s.pdf

Echeverri, J. (2009). Pueblos indígenas y cambio climático: el caso de la Amazonía colombiana. Bulletin de I'Institut Français d'Études Andines, 38 (1), pp. 13-28. ISSN 0303-7495.

Escobar, G y Berdegué, J. (1990). Tipificación de sistemas de producción agrícola. Santiago de Chile: RIMISP.

FAO-MGAP. TCP URU/3302. (2013). Clima de cambios: Nuevos desafíos de adaptación en Uruguay".

Farah, M. A. \& Pérez, E. (2003). Mujeres rurales y nueva ruralidad en Colombia. Cuadernos De Desarrollo Rural, (51). Recuperado a partir de https://revistas.javeriana.edu.co/index.php/desarrolloRural/article/view/1275

Figueroa-Bouriyú, A. (2018). Tecnologías apropiadas para el mejoramiento de la producción Ovino-Caprina, en el Resguardo Indígena de Provincial, Comunidad Las Tinajitas, Municipio de Barrancas La Guajira. Proyecto socio-comunitario para optar por el título de Zootecnista. Escuela de Ciencias Agrícolas, Pecuarias y del Medio Ambiente, Universidad Nacional Abierta y a Distancia. Riohacha, La Guajira. 45 pp.

Forero, J.; Torres, L.; Lozano, P.; Durana, C.; Galarza, J.; Corrales, E. \& Rudas, G. (2002). Sistemas de producción rurales en la región Andina colombiana. Análisis de su viabilidad económica, ambiental y cultural. Colciencias - Pontificia Universidad Javeriana. Bogotá, DC.

Fuenmayor, N.; Gamboa, T.; Acurero, L. (2004). La experiencia cooperativa en la cría de ovinos y caprinos en la Guajira venezolana. Cayapa. Revista Venezolana de Economía Social [en linea]. 4(7), 91-106. ISSN: 13175734. Disponible en: https://www.redalyc.org/articulo.oa?id=622/62240706

García, J. (2011). Pensar Sistémico. Centro Editorial Javeriano. Bogotá D.C., Colombia.

García, V. (s.f.). Estrategias adaptativas y amenazas climáticas. Centro de Investigaciones y Estudios Superiores en Antropología Social.

Geilfus, F. (2002). 80 herramientas participativas para el desarrollo participativo. Instituto Interamericano de Cooperación para la agricultura (IICA). ISBN13: 99923-7727-5. San José, Costa Rica.

Giménez, A.; Castaño, J.; Baethgen, W. \& Lanfranco, B. (2009). Cambio climático en Uruguay, posibles impactos y medidas de adaptación en el sector agropecuario. Serie Técnica № 178. ISBN: 978-9974-38-275-6. Uruguay.

Gimenez \& Lanfranco, B. (2012). Adaptación al cambio climático y la variabilidad: algunas opciones de respuesta para la producción agrícola en Uruguay. Revista Mexicana de Ciencias Agrícolas, (3) 611-620. Recuperado de http://www.redalyc.org/articulo.oa?id=263123205016

Gobernación de La Guajira. (2012). Plan de Desarrollo Departamento de La Guajira 2012- 2015 “La Guajira Primero". Riohacha. 360 pp. 
González, F. (1996). Ambiente y Desarrollo - Ensayos -: Reflexiones acerca de la relación entre los conceptos: Ecosistema, Cultura y Desarrollo. Pontificia Universidad Javeriana - IDEADE. Bogotá D.C.

Hammer $\varnothing$. Harper D. \& Ryan P. 2001. PAST: Paleontological Statistics Software Package for Education and Data Analysis. Paleontología Electrónica 4 (1): 1-9.

Hernández R.; Fernández, C. y Baptista, P. (2010), Metodología de la Investigación McGraw - Hill, México. D.F.

Herrera, F. (2013). Enfoques y políticas de desarrollo rural en México: Una revisión de su construcción institucional. Gestión y política pública, 22(1), 131-159. Recuperado en 05 de octubre de 2019, de http://www.scielo.org.mx/scielo.php?script=sci arttext\&pid=S1405$10792013000100004 \& \operatorname{lng}=e s \& t \operatorname{lng}=$ pt.

Herrera-Puebla, J., López-Seijas, T., Díaz-Pérez, Y., Cisneros-Zayas, E. \& Duarte-Díaz, C. (2017). Diagnóstico del recurso agua para la producción agrícola sostenible y compatible con el clima en el municipio Perico. Revista Ingeniería Agrícola, 7(2), 3-12.

Howden, S., Crimp A., Stokes C.J. (2008). Climate change and Australian livestock systems: Impacts, research and policy issues. Australian Journal of Experimental Agriculture 48, 780-788.

Ifejika, C. (2010). Drought Coping and Adaptation Strategies: Understanding Adaptations to Climate Change in Agro-pastoral Livestock Production in Makueni District, Kenya. European Journal of Development Research, 22(5), 623-642. doi:10.1057/ejdr.2010.39

Instituto Nacional de Estadística, Geografía e Informática - INEGI. (1994). Las unidades de producción rural VII censo agropecuario 1991. México. 179 pp.

Instituto Colombiano Agropecuario (ICA). (2015) Censo pecuario nacional. Recuperado de: http://www.ica.gov.co/getdoc/8232c0e5-b.e97-42bd-b07b9cdbfb07fcac/Censos2008.aspx

Instituto Colombiano Agropecuario - ICA. (s.f.). ICA. Programa nacional de ovinos/caprinos. Recuperado el 20 de abril de 2019, de https://www.ica.gov.co/areas/pecuaria/servicios/enfermedades-animales/especieovino-caprina.aspx

Instituto de Hidrología, Meteorología y Estudios Ambientales (IDEAM). (s.f.). Tiempo y clima. Recuperado de: http://www.ideam.gov.co/web/tiempo-y-clima/clima.

- Dirección General para la Prevención y Atención de Desastres (IDEAM-DGPAD). (2002). Efectos naturales y socioeconómicos del fenómeno El Niño en Colombia. Bogotá.

. (2015). Nuevos Escenarios de Cambio Climático para Colombia 2011- 2100 Herramientas Científicas para la Toma de Decisiones - Enfoque Nacional - Departamental: Tercera Comunicación Nacional de Cambio Climático. Recuperado de: http://documentacion.ideam.gov.co/openbiblio/bvirtual/022964/documento nacional departamental.pd $\underline{f}$

. (2018a). Cartas climatológicas - medias mensuales aeropuerto Almirante Padilla (Riohacha). Recuperado de: http://bart.ideam.gov.co/cliciu/rioha/tabla.htm

. (2018b). Datos mensuales de precipitación, evaporación, brillo solar, y temperaturas de la estación Almirante Padilla (Riohacha): periodo 2009-2016. Proporcionado por el Instituto de Hidrología Meteorología y Estudios Ambientales. 
IDEAM, PNUD, MADS, DNP, CANCILLERÍA. (2016). Inventario nacional y departamental de Gases Efecto Invernadero - Colombia. Tercera Comunicación Nacional de Cambio Climático. IDEAM, PNUD, MADS, DNP, CANCILLERÍA, FMAM. Bogotá D.C., Colombia. 73pp.

Instituto Geográfico Agustín Codazzi (IGAC). (2009). Estudio general de suelos y zonificación de tierras del departamento de La Guajira. Colombia

IPCC. (2014). Cambio climático 2014 Impactos, adaptación y vulnerabilidad Contribución del Grupo de trabajo II al Quinto Informe de Evaluación del Grupo Intergubernamental de Expertos sobre el Cambio Climático. Recuperado de: https://www.ipcc.ch/pdf/assessment- report/ar5/wg2/ar5 wgll spm es.pdf.

Jiménez, G.; Quechulpa, S.; Esquivel, E.; Soto, L.; Reyes, F.; Ruiz, M. \& Márquez, C. (2010). Ganadería y cambio climático: mitigación y adaptación en comunidades indígenas de Chiapas, México. LEISA Revista de agroecología. México.

Johnson, M. (1992). Lore: Capturing Traditional Environmental Knowledge. Ottawa, Dene Cultural Institute/IDRC. 190 pp.

Lara, R. \& Vides-Almonacid, R. (Eds). (2014). Sabiduría y Adaptación: El Valor del Conocimiento Tradicional en la Adaptación al Cambio Climático en América del Sur. UICN: Quito, Ecuador.

Maffesoli, M. (2005). La tajada del diablo. Compendio de subversión posmoderna. Siglo XXI Editores. México D. $\mathrm{F}$.

Manjarres, F. (2017). Plan de empresa para la producción de cordero en pie en la finca "Magalote" (Fonseca Guajira). Trabajo de grado para optar al título de administrador de empresas agropecuarias. Facultad de Ciencias Agropecuarias. Universidad La Salle. Bogotá. 103 pp.

Mastrandrea, T.E. Bilir, M. Chatterjee, K.L. Ebi, Y.O. Estrada, R.C. Genova, B. Girma, E.S. Kissel, A.N. Levy, S. MacCracken, P.R. Mastrandrea y L.L. White (eds.)]. Organización Meteorológica Mundial, Ginebra, Suiza, 34 págs.

March, I.; Cabral, H. \& Echeverria, Y. (2010). Una metodología para diseñar estrategias y planes de acción orientados a la adaptación del cambio climático para la conservación de biodiversidad, ecosistemas y servicios ecosistémicos.

Márquez, J. (2006). Protección a la Producción de Caprinos en La Guajira. Instituto Colombiano Agropecuario. ICA. Colombia.

Mejía, P. (2011). Situación sociolingüística del Wayuunaiki: Ranchería ElＰasito/Wayuunaiki Sociolinguistic Situation: Pasito Settlement. (Tesis maestría) Universidad Nacional de Colombia. Bogotá D.C.

Montealegre, J. \& Pabón, J. (2000). La Variabilidad Climática Interanual asociada al ciclo El Niño-La NiñaOscilación del Sur y su efecto en el patrón pluviométrico de Colombia. Meteorol. Colomb. 2:7-21. ISSN 0124-6984. Bogotá, D.C. - Colombia.

Montealegre, J. (2009). Estudio de la Variabilidad climática de la precipitación en Colombia asociada a procesos oceánicos y atmosféricos de meso y gran escala. Nota Técnica IDEAM, IDEAM -METEO/022-2009, Bogotá D.C. 
. (2014). Actualización del componente meteorológico del modelo institucional del IDEAM sobre el efecto climático de los fenómenos El Niño y La Niña en Colombia como insumo para el Atlas Climatológico. Informe Final. Instituto de Hidrología, Meteorología y Estudios Ambientales (IDEAM), Bogotá, Colombia.

Montecinos, C. (1999). Todos lo sabemos (o deberíamos saberlo). Monitor de Biotecnología y Desarrollo, Compendio 1995-1997. p. 45-46.

Moreno, D., \& Grajales, H. (2017). Caracterización de los sistemas de producción ovinos de trópico alto en Colombia: Manejo e indicadores productivos y reproductivos. Rev Vet Zoot., 64(3), 36-51.

Morton, J. (2007). The impact of climate change on smallholder and subsistence agriculture. PNAS 104: 1969719704.

Mussetta,P. \& Barrientos, M. (2015). Vulnerabilidad de productores rurales de Mendoza ante el Cambio Ambiental Global: clima, agua, economía y sociedad. Rev. FCA UNCUYO. 2015. 47(2): 145-170. ISSN impreso 0370-4661. ISSN (en línea) 1853- 8665

Neiman, G. \& Quaranta, G. (2006). Los estudios de caso en la investigación sociológica, parte 1, 213 pp. En: Vasilichis de Galindo, Irene. Estrategias de investigación cualitativa. Barcelona: Editorial Gedisa.

Organización de las Naciones Unidas para la Alimentación y la Agricultura (FAO). (2012). El estado mundial de la agricultura y la alimentación: invertir en la agricultura para construir un estado mejor. Viale delle Terme di Caracalla. Roma - Italia.198 p.

. (s.f.). Ganadería. Oficina Regional de la FAO para América Latina y el Caribe. Recuperado de: http://www.fao.org/americas/perspectivas/ganaderia/es/

Organización de las Naciones Unidas para la Educación, la Ciencia y la Cultura (UNESCO). (2015). Sistemas de Conocimientos Locales e Indígenas. Recuperado de: http://www.unesco.org/new/es/naturalsciences/priority-areas/links/related- information/what-is-local-and-indigenous-knowledge/

Organización Meteorológica Mundial (OMM). (2009). Tercera conferencia mundial sobre clima (CMC-3). Recuperado de: http://www.wmo.int/wcc3/documents/WCC 3_Statement_07-09-09_mods.pdf.

Ortiz, R. (2012). El cambio climático y la producción agrícola. Notas Técnicas \# ESG-TN-383 Banco Interamericano de Desarrollo.

Oyhantçabal, W., Vitale, E., \& Lagarmilla, (2010). P. El cambio climático y su relación con las enfermedades animales y la producción animal. Conf. OIE 2010, 169-177-

Pabón, J. \& Chaparro, R. (1998). Colombia en el ambiente global. Instituto de Hidrología, Meteorología y Estudios Ambientales. En: El Medio Ambiente en Colombia. 18-37p. Instituto de Hidrología, Meteorología y Estudios Ambientales, Bogotá. Panel Intergubernamental sobre Cambio Climático (IPCC). (2007). Cambio climático 2007.

Parra, W. (2011). La racionalidad campesina frente a la producción agropecuaria en el municipio de Garagoa (Tesis maestría en Desarrollo Rural). Pontificia Universidad Javeriana. Bogotá D.C.

Pelekais, C; Seijo, C; Neuman, N y Trompo, L (2014) El ABC de la Investigación Guía Didáctica, Sexta Edición. Ediciones Astro Data. Maracaibo Venezuela) 
Pinilla-Herrera, M.; Rueda, A.; Pinzón, C. \& Sánchez, J. (2012). Percepciones sobre los fenómenos de variabilidad climática y cambio climático entre campesinos del centro de Santander, Colombia. Ambiente y Desarrollo, ISSN-e 0121-7607, Vol. 16, №. 31.

Poveda, G. (2004). La hidro-climatología de Colombia: Una síntesis desde la escala interdecadal hasta la escala diurna. Revista de la Academia Colombiana de Ciencias, 28(107), 201-222.

Presidencia de la República -Consejo Nacional de Política Social. (2017). Estrategia para el desarrollo integral de La Guajira. Bogotá: Dirección Nacional de Planeación.

Programa de adaptación al cambio climático TNC-Región México y Norte de Centroamérica. The nature conservanccy. México.9p.

Programa de las Naciones Unidas para el desarrollo (PNUD). (2010). Segunda Comunicación Nacional ante la Convención Marco de las Naciones Unidas sobre Cambio Climático. Editorial Scripto Ltda. Bogotá Colombia. 437 p.

. (2012). El Caribe colombiano frente a la gestión integral del riesgo y la adaptación al cambio climático. Proyecto: Fortalecimiento de las capacidades institucionales para la implementación de prácticas locales de gestión del riesgo como medida de adaptación al cambio climático en la zona insular y costera del Caribe colombiano. ISBN. 978-958-8447-82-7. Enero de 2012. Recuperado de: http://www.pnud.org.co/2012/cartilla_guajira.pdf

Informe de síntesis. Contribución de los Grupos de trabajo I, II y III al Cuarto Informe de evaluación del Grupo Intergubernamental de Expertos sobre el Cambio Climático [Equipo de redacción principal: Pachauri, R.K. y Reisinger, A. (directores de la publicación)]. IPCC, Ginebra, Suiza.104 pp.

(2007). Climate Change 2007: Impacts, Adaptation and Vulnerability. Contribution of Working Group II to the Fourth Assessment Report of the Intergovernmental Panel on Climate Change. Cambridge University Press, Cambridge, UK y Nueva York. Disponible en: http://www.ipcc.ch/publications_and_data/ar4/ wg2/en/contents. html [consulta: 19- 6-2017].

. (2014). Cambio climático 2014: Impactos, adaptación y vulnerabilidad-Resumen para responsables de políticas. Contribución del Grupo de trabajo II al Quinto Informe de Evaluación del Grupo Intergubernamental de Expertos sobre el Cambio Climático [Field, C.B., V.R. Barros, D.J. Dokken, K.J. Mach, M.D.

Pulido, J. (2015). Aproximación al Análisis de Ciclo de Vida Social (ASCV) para la fase de producción de Caféen el municipio deViotá, Cundinamarca(Colombia). Bogotá: Facultad de Ciencias AmbientalesPrograma en Administración y Gestión Ambiental.

Quintero-Angel, M. \& Carvajal-Escobar, Y. (2010). Adaptación o mitigación al cambio climático: Consideraciones en el contexto latinoamericano. Investigación, Biodiversidad y Desarrollo 2010; 29 (2). 123-32 pp.

Quintero, A.; Walkiria, C.; Fernández, L. \& De Calzadilla, J. (2008). Análisis multivariado de indicadores socioeconómico-productivos del ganado caprino-ovino en el departamento de La Guajira, Colombia. Revista de la Universidad Francisco de Paula Santander. ISSN 0122-820X, Vol. 2, año 13.

. (2010). Diagnóstico del sistema de producción-comercialización del ganado caprino-ovino en el departamento de La Guajira, Colombia. Aplicación del Escalamiento Óptimo. Revista Ciencias Técnicas Agropecuarias, 19(2), 57-64. 
Ramírez, K. (2007). Desde el desierto. Notas sobre paramilitares y violencia en territorio Wayuu de la media Guajira. Colección Wounmainkat Nº 1. ISBN. 987-958-44-1682-7.

Redacción Estilo de Vida. Niño reduce lluvias en plena temporada invernal. En El Tiempo, Bogotá, mayo 6 de 2015. Recuperado de: http://www.eltiempo.com/estilo-de-vida/ciencia/nino-reduce-lluvias-encolombia/15693319

Riohacha, D.E.T y C. (2016). Plan de Desarrollo Distrital Riohacha incluyente y sostenible 2016-2019. 301 pp.

Rojas, A. (2005). Proyecto Ecoandino. Tomo I. Conceptos y Metodología. Capítulo 8. Territorio y ordenamiento ambiental. Unidad de Parques Nacionales Naturales de Colombia. Plan Mundial de Alimentos (PMA) de las Naciones Unidas. Bogotá. Colombia.

Rojas, J. (2008). La Agenda Territorial del desarrollo rural en América Latina. Observatorio de la Economía Latinoamericana, no 96. Recuperado de: http://www. eumed.net/cursecon/ecolat/la/ 08/jrl.htm

Roncallo F., Toloza P., Barros H., Silva Z., Avila M., Araujo G., Robledo, L. (1999). Caracterización tecnológica de las explotaciones caprinas y ovinas en los departamentos de La Guajira, Cesar y Magdalena. Corpocaribe (Colombia) v. 2 (2) p. 5-15.

Rosnay, D. (1977). La revolución sistémica una nueva cultura. En D. Rosnay, El Macroscopio: Por una Visión Global, pp. 72 - 118. Madrid, España: AC Libros Científicos y Técnicos.

Rúa, C. (2015). Evaluación del efecto del sistema de producción sobre el consumo de alimento y la producción de leche en cabras Saanen y Alpina (Tesis maestría). Universidad de Antioquia. Medellín.

Ruíz, A. \& Pabón, J. (2013). Efecto de los fenómenos de El Niño y La Niña en la precipitación y su impacto en la producción agrícola del departamento del Atlántico (Colombia). En: Cuadernos de geografía. Revista colombiana de geografía. Vol. 22, № 2, jul.-dic. de 2013. ISSN 0121-215X (impreso). Bogotá, Colombia, pp. 35-54

Salazar-Romero, I. (2017). Viabilidad y factibilidad para la creación de una granja productora de ovinos en La Guajira. Trabajo de grado para optar al título de Médico Veterinario y Zootecnista. Facultad Ciencias de la Salud, Medicina Veterinaria y Zootecnia. Universidad Tecnológica de Pereira. Pereira, Risaralda. 41 pp.

Saraceno, E. (2001). La experiencia europea de desarrollo rural y su utilidad para el contexto latinoamericano. Seminario Internacional "Agricultura Familiar y Desarrollo Sustentable". 10 pp. EMBRAPA. Brasilia.

Scalone, M. (2007). El Enfoque de Sistema, Sistema de producción Agropecuario, Sistemas Agrarios Regionales. Montevideo, Uruguay: Instituto de Agrimensura.

Seo, S. \& Mendelsohn, R. (2007b): The impact of climate change on livestock management in Africa: a structural Ricardian analysis. In: Policy Research Working Paper 4603, Washington - USA.

Sepúlveda, S., Rodríguez, A., Echeverri, R., Portilla, M. (2003). El enfoque territorial del desarrollo rural. San José de Costa Rica. Ed. IICA.

Smit, B. \& Wandel, J. (2006). Adaptation, adaptive capacity and vulnerability. Global Environ Chang. 16(3), pp.282- 92.

Stephenson, J. (2010). Livestock and climate policy: less meat or less carbon? Round Table on Sustainable Development (24 February 2010). SG/SD/RT (2010)1.OECD (Organisation for Economic Co-operation and Development). 
Steward, J. (1973). Theory of Culture Change. The Methodology of Multilinear Evolution. 2a. ed., University of Illinois Press, EE.UU.

Stoińska-Kairska I. (2017). Del sueño a la eternidad. Ritos funerarios en la Guajira venezolana. Etnografia. Praktyki, Teorie, Doświadczenia 2017(3), 55-189.

Timaure-Jiménez, C., Pozo, J. A., Soto-Ysea, \& Y., Guerere-Morales, A. (2015). Sistemas de producción caprina y ovina en la subregión Costa Oriental del Lago de Maracaibo. Tecnología en Marcha, 28(1), 71-90.

Torres Guevara, J. (Octubre 2014 - Enero 2015 de 2015). Experiencias de adaptación al cambio climático, los conocimientos ancestrales, los conocimientos contemporáneos y los escenarios cualitativos en los Andes. Alcances y límites (Perú). Apuntes de Investigacción, nd.

Ulloa, A. (2008). "Implicaciones ambientales y culturales del cambio climático para los pueblos indígenas", en: Ulloa, Astrid; Escobar, Elsa Matilde; Donato, Luz Marina y Escobar, Pía (eds.): Mujeres indígenas y Cambio Climático. Perspectivas Latinoamericanas, Bogotá: Universidad Nacional de Colombia, Fundación Natura, UNODC, 17-34.

. (2011). "Repensando la naturaleza. Encuentros y desencuentros disciplinarios en torno a lo ambiental" En: Colombia. Ed. ICANH ISBN: 958-8181-02 v. 1 págs.246 Palabras: Ambiente, Historia de la Amazonia, Naturaleza, Áreas: Ciencias Humanas -- Historia, Sectores: Educación.

. (2012). Working Paper, No. 21, 2012. Producción de conocimientos en torno al clima. Procesos históricos de exclusión/apropiación de saberes y territorios de mujeres y pueblos indígenas. Recuperado de: http://edocs.fuberlin.de/docs/servlets/MCRFileNodeServlet/FUDOCS_derivate_000000001986/21_WP_Ulloa_online.pdf? hosts=local

Unidad Nacional para la Gestión del Riesgo de Desastres (UNGRD), Alcaldía de Riohacha, Fundación Alpina. (2014). Seguridad alimentaria y nutricional Fortalecimiento de la actividad productiva ovino-caprina y de la producción agrícola en las comunidades indígenas Wayuu afectadas por la ola invernal. Primera edición. Bogotá, 86 pp.

Vargas-López, S., Bustamante-González, A., Zaragoza, J., Morales-Jiménez, J., Vargas-Monter, J. (2018). Estrategias de adaptación de las unidades de producción ganaderas a los riesgos climáticos. Agroproductividad 11(2), 75-80.

Vargas, S. (2003). Análisis y desarrollo del sistema de producción agrosilvopastorilcaprino para carne en condiciones de subsistencia de Puebla, México (tesis doctoral). Universidad de Córdoba. Córdoba - España. $237 \mathrm{pp}$.

World Wildlife Fund for Nature (WWF). (2013). Nodos regionales de cambio climático. Reporte consolidado de línea base análisis de riesgos climáticos y necesidades de adaptación climática. Cali, Colombia. 141 pp.

Yin, R. (1994). Case Study Research. Design and Methods, Applied Social Research Methods Series, vol. 5, second edition. Sage Publications, London.

Esta obra está bajo una Licencia Creative Commons Attribución-NoCommercial 4.0 International

(cc) BY-NC 